\title{
Plant Taxonomy
}

National Cancer Institute

\section{Source}

National Cancer Institute. Plant Taxonomy. NCI Thesaurus. Code C19058.

The systematic classification of plants. 